\title{
TORSIONAL OSCILLATIONS AND THE SOLAR DYNAMO REGIME
}

\author{
I. TUOMINEN ${ }^{1}$, G. RÜDIGER ${ }^{2}$, A. BRANDENBURG ${ }^{1}$ \\ ${ }^{1}$ Observatory and Astrophysics Laboratory, University of Helsinki \\ Tähtitorninmäki, SF-00130 Helsinki, Finland \\ ${ }^{2}$ Sternwarte Babelsberg, Rosa-Luxemburg-Str. 17a, \\ 1591 Potsdam, German Democratic Republic
}

\begin{abstract}
We discuss the observational results of cyclic variations of solar rotation and how these can be used as a means of probing the solar dynamo. We shortly describe two examples of dynamo models where the $\alpha$-effect has been modified, and compare the resulting flows to the observations.
\end{abstract}

\section{Introduction}

The observed temporal dependence of the solar differential rotation can be understood as an empirical constraint to the deep-seated turbulent dynamo. In our opinion the latter forms the physical background for all the various cyclic activity phenomena of the main sequence stars. The construction of the stellar dynamo can only be calibrated by the Sun, which is the best observable star. As only surface features are observed, however, it is not a trivial task to solve the inverse problem of finding either the inner flow or field regime. We continue here to probe the dynamo by means of the cyclic variations of the solar rotational rate, $\Omega=\Omega(\theta, t)$, in addition to the butterfly diagram and the magnetic polar branch. As we have described in much detail (Rüdiger et al., 1986, hereinafter referred to as paper I) all the polynomial modes in the series expansion

$$
\Omega=\Omega_{0} \sum_{n=1,3, \ldots} \omega_{n} P_{n}^{1}(\cos \theta) / \sin \theta
$$

proved to be periodically time-dependent. The modes with $n>5$, i.e. the original torsional oscillation (Howard and LaBonte, 1980), form a 4-belt pattern in both hemispheres migrating in 22 years from the poles to the equator. Two belts are faster ("accelerating") and two are slower ("decelerating") than the large-scale modes. The main acceleration belt lies always equatorwards from the centre of the belt of magnetic activity. The pattern starts its migration at the poles during the activity minimum - and reaches, after 22 years, the equator also at the minimum. Additionally, also the modes with $n=3$ and $n=5$ vary in phase with each other while their maxima occurs at maximal activity. The full pattern of variable $\Omega$ looks somewhat different (Snodgrass and Howard, 1985). There is a speeding up of the rotation at high latitudes during and just after the activity maximum and a wave then emerges at mid-latitudes during the minimum and moves toward the equator. Compared 
with the temporal variation of the large-scale modes $(n<7)$, the torsional oscillation is the dominating effect, the maximum contribution obtained from the mode with about $n=9$. A similar power law has recently been derived by Stenflo (1988) for the contribution of the polynomial modes of the radial magnetic field over the cycle. Approximately according to the expectations (the induced flow behaves quadratically with respect to the magnetic field) it was found that the polynomials with $n=5$ and $n=7$ provide the largest contribution to the solar radial magnetic field. This finding is also an important empirical constraint for an optimal dynamo model.

These observations in mind we recall the results of Paper $I$ in which the first published solar-type dynamos (Steenbeck and Krause, 1969) were analyzed by means of the solution of the Navier-Stokes equation with mean-field Lorentz force. What we found was that indeed two accelerating and two decelerating belts of $\Omega$ exist for dynamos with a relatively deep convection zone. Also the location of the lower fast belt was correct and even the other mentioned properties of $\Omega(\theta, t)$ could be reproduced, besides the behaviour of $\omega_{3}$ which varied in anti-phase relative to $\omega_{5}$. As the old dynamo models were only tractable in a very rough way and as new ideas on the inner profile of the $\alpha$-effect have meanwhile been formulated, we analyze with some recently established dynamo models (Brandenburg and Tuominen, 1988; Brandenburg, 1989) their associated magnetically induced mean flows. We adopt the theoretical formalism of Paper I, also with its shortcomings described there in detail. We mainly change the two most important input parameters of the dynamo theory, i.e. the $\alpha$-effect and rotation law. With respect to the latter it was the helioseismologically derived solar rotation law which is involved, so that both the radial and the latitudinal gradient of $\Omega$ contribute as inducing effects to the dynamo action. The fixing of $\alpha$ is more troublesome. It is certainly positive near the surface due to the dominating density gradient,

$$
\alpha \approx \Omega \ell_{\rho},
$$

where $\ell_{\rho}=(d \ln \rho / d r)^{-1}$ is the density scale height. But deep in the convection zone the opposite velocity gradient may weaken its value until even the sign is changed,

$$
\alpha \approx \Omega \ell_{\rho}\left(1-\ell_{\rho} / \ell_{u}\right)
$$

with $\ell_{u}=\left(d \ln u_{\text {turb }} / d r\right)^{-1}$ (Krause, 1967). This consideration may especially hold in the overshoot region below the convectively unstable zone as the density scale length $\ell_{\rho}$ becomes larger than $\ell_{u}$ (cf. Pidatella and Stix, 1986).

\section{Dynamos with negative $\alpha$}

We have thus analyzed first a model with a lower region with a negative $\alpha$-effect (cf. Fig. $2 b$ in Glatzmaier, 1985). The results for this model are given in Fig. 1. The butterfly diagram indeed exhibits a very solar-like behaviour. The toroidal field belts start at latitude $60^{\circ}$ and reach maximum at $15^{\circ}$. The phase relation between $B_{r}$ and $B_{\phi}$, however, does not agree as both components are varying in phase, in opposite to observations (Stix, 1976). Indeed, as a consequence the accelerarion belt is located above the aztivity zone, contrary to the observations. Furthermore, $\omega_{1}$ varies much too strongly. That clearly reveals the existence of too large contributions of the lower polynomial modes in the magnetic field representation 
(cf. Stenflo, 1988). On the other hand, also the other modes in Eq. (1) have "wrong" properties: $\omega_{3}$ and $\omega_{5}$ and are out of phase. We have thus to state serious contradictions between the model and the real observations, in particular of the time-dependence of the rotational rate (1). Similar difficulties have been described by Glatzmaier (1986) for just this type of dynamo.
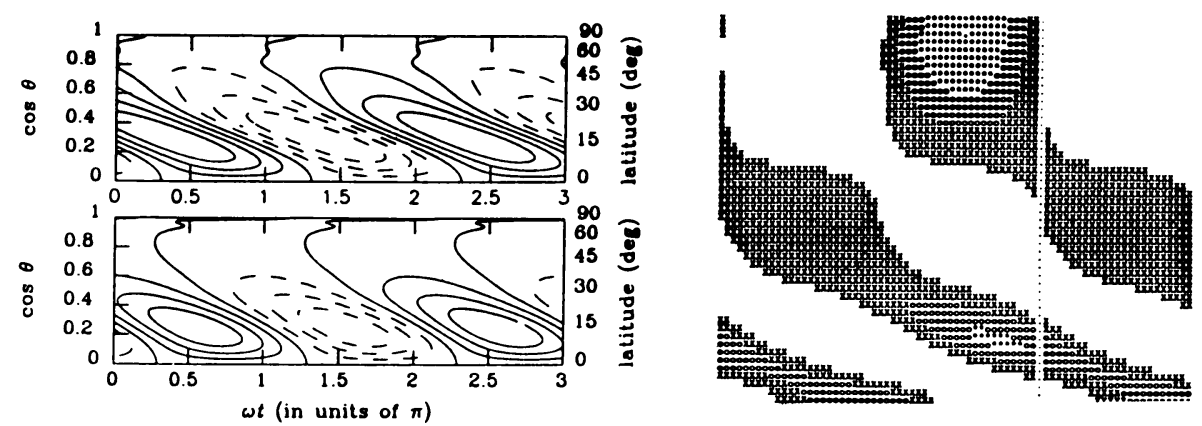

Fig. 1. Left panel: Butterfly diagrams for the $B_{r}$ - and $B_{\phi}$-field for the dynamo model with negative $\alpha$. Right panel: The corresponding torsional wave pattern with $n>5$. Note that in the right panel the ordinate is linear in $\theta$ and the abscissa goes from 0 to $1.5 \pi$. The shaded areas define faster rotation.

\section{Higher order terms in $\alpha$}

We suggest thus to reject the idea of negative $\alpha$ at the bottom of the convection zone and turn to another modification of the traditional $\alpha$-profile. As is well known from the theory of differential rotation, it is not enough to consider only the turbulence correlations which are linear in the rotation rate $\Omega$. As the Sun is not a slow rotator in the sense of the turbulence theory ("slow" means $\tau_{\text {rot }} \ll \tau_{\text {corr }}$ ) also higher order terms must be involved in the calculations (cf. Rüdiger, 1989). The same arguments, of course, hold for the $\alpha$-effect: Higher order terms also exist with quite another latitude dependence,

$$
\alpha=\alpha_{0}-\alpha_{2} \cos ^{2} \theta
$$

(Rüdiger, 1980, Schmitt, 1987), so that it even may change its sign at some latitude. For $\alpha_{0}=\alpha_{2}$ the $\alpha$-effect vanishes at the poles. We want to favour this case rather than that where there is a node at a certain latitude, e.g. at $30^{\circ}$ as Schmitt suggests, which seems to be too extreme. Our choice only concentrates the $\alpha$-effect to lower latitudes instead having maximum at the pole. The hydrodynamical results of this procedure are rather surprising (Fig. 2). The butterfly diagram and polar branch have the right properties and also the phase relation agrees with the observations, i.e. $B_{r} B_{\phi}<0$. In addition the Stenflo constraint is fulfilled, i.e. the lowest modes do not contribute too strongly. The torsional oscillation belts have the correct location in the latitude-time-diagram, compared to the magnetic field, but they start at the activity maximum (reaching the equator after a whole 22-year cycle also at the maximum). That is a first contradiction while the second one 
is the out-of-phase variation of $\omega_{3}$ and $\omega_{5}$. But the behaviour of $\omega_{3}$ itself corresponds to the observations, it has maximum at the activity maximum and minimum at the activity minimum. Obviously, only the term $\omega_{3}$ is still out of correspondence.
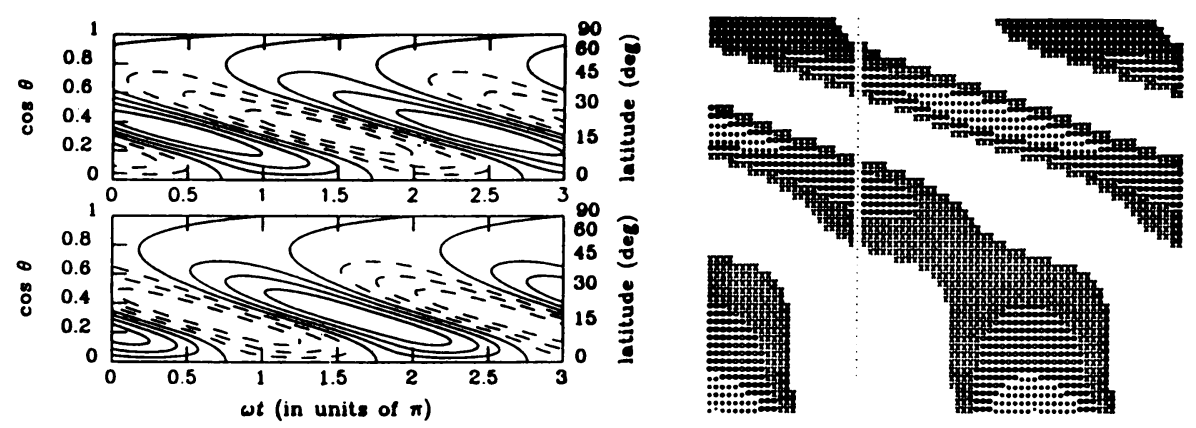

Fig. 2. Left panel: Butterfly diagrams for the $B_{r}$ and $B_{\phi}$-field for the dynamo model with $\alpha_{0}=\alpha_{2}$. Right panel: The corresponding torsional wave pattern.

We understand these examples as an improvement of the preliminary analysis presented in Paper I, where only the depth of the convection zone resulted from the comparison of the calculations and the observations. It seems possible to use the observed flow pattern as a precise tool for analyzing the inner turbulent regime of stellar convection.

\section{References}

Brandenburg, A.: 1989, Dissertation, Univ. of Helsinki.

Brandenburg, A., Tuominen, I.: 1988, Adv. Space Sci., 8, (7)185.

Glatzmaier, G.A.: 1985, Astrophys. J. 291, 300.

Glatzmaier, G.A.: 1986, Geophys. Astrophys. Fluid Dyn. 31, 137.

Howard, R., LaBonte, B.J.: 1980, Astrophys. J. 239, L33.

Krause, F.: 1967, Habilitationsschrift, University of Jena.

Pidatella, R. M., Stix, M.: 1986, Astron. Astrophys. 157, 338.

Rüdiger, G.: 1980, Geophys. Astrophys. Fluid Dyn. 16, 239.

Rüdiger, G.: 1989, Differential rotation and Stellar Convection:

Sun and Solar-type stars, Gordon and Breach, New York.

Rüdiger, G., Tuominen, I., Krause, F., Virtanen, H.: 1986, Astron.

Astrophys. 166, 306.

Schmitt, D.: 1987, Astron. Astrophys. 174, 281.

Snodgrass, H. B., Howard, R.: 1985, Science 228, 945.

Steenbeck, M. and Krause, F.: 1969, Astron. Nachr. 291, 49.

Stenflo, J.O. : 1988, Astrophys. Spa. Sci. 144, 321.

Stix, M.: 1976, Astron. Astrophys. 47, 243. 